\title{
Avaliação das propriedades termodinâmicas e físico-químicas do mesocarpo do maracujá quando submetido aos processos combinados de secagem
}

Evaluation of the thermodynamic and physicochemical properties of passion fruit mesocarp when submitted to combined drying processes

Evaluación de las propiedades termodinámicas y fisicoquímicas del mesocarpio del maracuyá al someterlo a procesos de secado combinados

Recebido: 18/09/2021 | Revisado: 30/09/2021 | Aceito: 04/10/2021 | Publicado: 07/10/2021

Fabrícia Santos Andrade ORCID: https://orcid.org/0000-0001-6280-5013 Universidade Federal de Campina Grande, Brasil

E-mail: fabricia.santo.andrade@ hotmail.com

Pablícia Oliveira Galdino

ORCID: https://orcid.org/0000-0001-6996-0550 Universidade Estadual da Paraíba, Brasil

E-mail: pabliciaog@hotmail.com

Ângela Maria Santiago

ORCID: https://orcid.org/0000-0001-7108-6890 Universidade Estadual da Paraíba, Brasil

E-mail: angelamariasantiago01@gmail.com

Francyellen Beserra de Oliveira

ORCID: https://orcid.org/0000-0002-7368-4293 Universidade Estadual da Paraíba, Brasil

E-mail: francyellen.oliveira2014@gmail.com

Marcello Maia de Almeida

ORCID: https://orcid.org/0000-0001-7889-126X Universidade Estadual da Paraíba, Brasil

E-mail: Marcello_maia2000@yahoo.com.br

Plúvia Oliveira Galdino

ORCID: https://orcid.org/0000-0001-5286-7114 Universidade Federal de Campina Grande, Brasil

E-mail: pluviagaldino@hotmail.com

Mércia Melo de Almeida Mota

ORCID: https://orcid.org/0000-0002-1336-9355

Universidade Federal de Campina Grande, Brasil

E-mail: mercia01@gmail.com

Maria Celly dos Santos Silva

ORCID: https://orcid.org/0000-0001-5844-4083

Universidade Estadual da Paraíba, Brasil

E-mail: mariacelly018@gmail.com

Maciel de Lima Silvestre

ORCID: https://orcid.org/0000-0001-7492-401X

Universidade Estadual da Parába, Brasil

E-mail: limamacie164@gmail.com

Marina Gonçalves da Silva Nascimento

ORCID: https://orcid.org/0000-0001-9053-5719

Universidade Estadual da Paraíba, Brasil E-mail: marigdsn@gmail.com

\section{Resumo}

A combinação de métodos de secagem tem sido apontada como alternativa econêmica e segura para a conservação de produtos alimentícios. Portanto, o presente estudo teve como objetivo realizar os processos combinados de osmodesidratação e secagem convectiva complementar do mesocarpo do maracujá, ajustar modelos matemáticos e avaliar a influência desses processos nas suas propriedades termodinâmicas e físicoquímicas. Um planejamento experimental $2^{2}$ foi realizado para avaliar a influência da concentração de sacarose e da temperatura, sobre a perda de água, perda de massa e ganho de sólidos na desidratação osmótica. $\mathrm{O}$ experimento selecionado conforme o planejamento foi-seco em estufa nas temperaturas de 50,60 e $70^{\circ} \mathrm{C}$, por meio dos dados foram obtidas as propriedades termodinâmicas do processo. A melhor condição de processo durante a desidratação osmótica foi a $60{ }^{\circ}$ Brix e $60{ }^{\circ} \mathrm{C}$. O modelo matemático Logarítmico foi o que melhor se ajustou aos dados experimentais da cinética de secagem e o processo de 
secagem foi definido como endorgênico e não espontâneo. Mesmo ocorrendo variações nos parâmetros físicos e físicoquímicos após a desidratação osmótica seguida de secagem convectiva complementar, torna-se viável o aproveitamento desse resíduo como forma alternativa na alimentação, além de minimizar os impactos ambientais causados quando lançados no meio ambiente.

Palavras-chave: Difusividade; Entalpia; Logarítmico; Resíduos; Sacarose.

\begin{abstract}
The combination of drying methods has been identified as an economical and safe alternative for the conservation of food products. Therefore, the present study aimed to perform the combined osmodhydration processes and complementary convective drying of the passion fruit mesocarp, adjust mathematical models and evaluate the influence of these processes on their thermodynamic and physical-chemical properties. An experimental design $2^{2}$ was carried out to evaluate the influence of sucrose concentration and temperature, on water loss, mass loss and solids gain on osmotic dehydration. The experiment selected as planned was kiln dried at temperatures of 50, 60 and $70{ }^{\circ} \mathrm{C}$, using the data to obtain the thermodynamic properties of the process. The best process condition during osmotic dehydration was at $60{ }^{\circ}$ Brix and $60{ }^{\circ} \mathrm{C}$. The Logarithmic mathematical model was the one that best fit the experimental data of the drying kinetics and the drying process was defined as endorganic and not spontaneous. Even with variations in physical and physicalchemical parameters after osmotic dehydration followed by complementary convective drying, it is feasible to use this residue as an alternative form of food, in addition to minimizing the environmental impacts caused when released into the environment.
\end{abstract}

Keywords: Diffusivity; Enthalpy; Logarithmic; Residue; Sucrose.

\title{
Resumen
}

La combinación de métodos de secado se ha señalado como una alternativa económica y segura para la conservación de productos alimentarios. Por lo tanto, el presente estudio tuvo como objetivo realizar los procesos combinados de osmodesidratación y secado convectivo complementario del mesocarpio de maracuyá, ajustar los modelos matemáticos y evaluar la influencia de estos procesos en sus propiedades termodinámicas y físicoquímicas. Se realizó una planificación experimental 22 para evaluar la influencia de la concentración de sacarosa y la temperatura, en la pérdida de agua, la pérdida de masa y la ganancia de sólidos en la deshidratación osmótica. El experimento seleccionado según la planificación fue secado en horno a temperaturas de 50,60 y $70{ }^{\circ} \mathrm{C}$, a través de los datos se obtuvieron las propiedades termodinámicas del proceso. La mejor condición de proceso durante la deshidratación osmótica fue a $60{ }^{\circ} \mathrm{Brix}$ y $60{ }^{\circ} \mathrm{C}$. El modelo matemático logarítmico fue el que mejor se ajustó a los datos experimentales de la cinética de secado y el proceso de secado se definió como endógeno y no espontáneo. Aunque se produzcan variaciones en los parámetros físicos y fisicoquímicos después de la deshidratación osmótica seguida de un secado convectivo complementario, se hace factible el uso de este residuo como forma alternativa en los alimentos, además de minimizar los impactos ambientales causados al ser liberado en el medio ambiente.

Palabras clave: Difusividad; Entalpía; Logaritmo; Residuos; Sacarosa.

\section{Introdução}

O Brasil se destaca como o maior produtor e consumidor mundial de maracujá (Passiflora edulis Sims), em 2018 produziu 602.651 toneladas, correspondendo a uma área de 42.731 ha (14.10 t ha ${ }^{1}$ ), dos quais $62.3 \%$ estão na Região Nordeste, sendo o estado da Bahia com $26.7 \%$ da produção nacional (IBGE, 2019). É um fruto nativo da Caatinga com potencial para uso pelas indústrias alimentícia e farmacêutica (Costa et al., 2020). Esta espécie é cultivada em mais de 95\% dos Pomares brasileiros, principalmente devido à sua qualidade, produtividade, vigor e rendimento de suco (Cavalcante et al., 2019; IBGE, 2019; Lima et al., 2020).

A casca (mesocarpo) do maracujá é um coproduto da produção industrial de sucos e seu peso corresponde a aproximadamente $90 \%$ da fruta fresca que é descartada como resíduo durante o processamento. Vários estudos apontam uma expressiva quantidade de pectina contida no mesocarpo do maracujá e sua conversão em produtos, oferecendo uma grande oportunidade para seu uso (Kulkarni \& Vijayanand, 2010; Nascimento, Calado \& Carvalho, 2012; Rotta et al., 2020).

A desidratação osmótica, embora possa ser implementada como um processo autônomo, geralmente é utilizada como pré-tratamento para alguns tipos de secagem subsequente. Além de atenuar alguns dos efeitos indesejáveis causados pela secagem por convecção, a desidratação osmótica ajuda a remoção de água e a absorção de sólidos, permitindo a fabricação de produtos minimamente processados. Como não requer alterações de fase aquosa no decorrer do processo, possui considerável 
importância industrial quando se trata de eficiência energética (Panarese, Tylewicz, Santagapita, Rocculi \& Rosa, 2012; Aires, Aires, Silva Júnior \& Silva, 2016). Esta combinação de métodos de secagem tem sido apontada como alternativa econômica e segura para a conservação de produtos alimentícios, além de possibilitar a obtenção de produtos desidratados de melhor qualidade quando comparado aos produtos desidratados convencionalmente (Silva \& Pedro, 2018).

Neste contexto, o presente estudo teve como objetivo realizar os processos combinados de osmodesidratação e secagem convectiva complementar no mesocarpo do maracujá, ajustar modelos matemáticos e avaliar a influência desses processos nas suas propriedades termodinâmicas e físico-químicas.

\section{Método da Pesquisa}

Os maracujás (Passiflora edulis Sims) foram obtidos na cidade de Campina Grande, Paraíba e selecionados por seu estádio de maturação e qualidade visual. Os frutos inteiros foram submetidos a uma lavagem inicial, em seguida foram imersos em solução de hipoclorito de sódio 100 ppm por 5 minutos e por fim foram lavados novamente para remover o excesso dessa solução. Após o processo de sanitização, os maracujás foram descascados com o auxílio de uma faca de aço inoxidável, retirando o epicarpo, película amarela que recobre o fruto e assim despolpados, restando apenas o mesocarpo e a película interna à ele que foi retirada com o auxílio de uma colher de aço inoxidável, o mesocarpo foi cortado em fatias de 1,5 x 2,5 cm. As amostras foram branqueadas (imersas em água em ebulição por 5 minutos) com a finalidade de inativar as enzimas responsáveis pelo escurecimento.

\subsection{Planejamento experimental fatorial para desidratação osmótica}

Um planejamento experimental $2^{2}$ com 3 repetições no ponto central, totalizando 7 ensaios foi realizado para avaliar a influência e o comportamento experimental das variáveis independentes (variáveis de entrada), concentração de sacarose e temperatura, sobre as variáveis respostas (dependentes), perda de água (PA), perda de massa (PM) e ganho de sólidos (GS) na desidratação osmótica. A concentração de sacarose variou de (-1) $40{ }^{\circ} \mathrm{Brix}$ a $(+1) 60^{\circ} \mathrm{Brix}$ e a temperatura-de $(-1) 40{ }^{\circ} \mathrm{C}$ a $(+1)$ $60^{\circ} \mathrm{C}$. O efeito das variáveis independentes sobre as variáveis dependentes foi avaliado mediante análise estatística, utilizandose o programa computacional Statistica® versão 11.0.

\subsection{Desidratação Osmótica (DO)}

As soluções hipertônicas de sacarose nas concentrações de 40, 50 e $60^{\circ}$ Brix foram preparadas utilizando sacarose comercial e água destilada, adicionada de $30 \%$ de polpa do maracujá, para que as amostras ficassem saborizadas. Todo o processo de desidratação ocorreu em estufa com circulação de ar nas temperaturas de $\left(40,50\right.$ e $\left.60{ }^{\circ} \mathrm{C}\right)$. A solução de sacarose foi mantida em banho-maria até atingir a temperatura de processo $\left(40,50\right.$ e $\left.60^{\circ} \mathrm{C}\right)$, só então foi adicionada ao recipiente contendo as amostras até completa imersão das fatias.

A massa e o teor de água das fatias foram determinados periodicamente durante intervalos de 15 minutos, totalizando 90 minutos de desidratação osmótica. Para cada experimento do planejamento foi realizado o cálculo da perda de massa e do teor de água, segundo a metodologia descrita em Brasil (2008). A perda de massa (PM), a perda de água (PA) e o ganho de sólidos (GS), foram calculados através das Equações (1, 2 e 3), respectivamente.

$$
\begin{aligned}
& \operatorname{PM}(\%)=\frac{\left(\mathrm{M}_{0}-\mathrm{M}_{\mathrm{t}}\right)}{\mathrm{M}_{0}} \times 100 \\
& \operatorname{PM}(\%)=\frac{\left(\mathrm{Ma}_{0}-\mathrm{Ma}_{\mathrm{t}}\right)}{\mathrm{M}_{0}} \times 100
\end{aligned}
$$




$$
\mathrm{GS}(\%)=\mathrm{PA}(\%)-\mathrm{PM}(\%)
$$

onde: $\mathrm{M}_{0}$ - massa inicial do produto $(\mathrm{g}) ; \mathrm{M}_{\mathrm{t}}$ - massa do produto a um tempo $\mathrm{t}(\mathrm{g}) ; \mathrm{Ma}_{0}$ - teor de água no produto (g); e $\mathrm{Ma}_{\mathrm{t}}$ - teor de água no produto a um tempo $\mathrm{t}(\mathrm{g})$.

\subsection{Cinética de secagem}

As fatias do mesocarpo do maracujá na melhor condição do planejamento fatorial da desidratação osmótica foram colocadas em telas de aço inoxidável, e uniformemente espalhadas, formando uma camada fina. Para iniciar o experimento, as bandejas foram colocadas em um secador com circulação e velocidade de ar de $1.0 \mathrm{~m} / \mathrm{s}$, para a realização das cinéticas de secagem nas temperaturas de 50,60 , e $70^{\circ} \mathrm{C}$, no início e no final do processo foram determinadas as massas secas e calculados os teores de água de acordo com Brasil (2008). Por meio dos dados experimentais, foi possível calcular os valores da razão do teor de água (Equação 4).

$$
\mathrm{RX}=\frac{\mathrm{X}_{\mathrm{bs}}-\mathrm{X}_{\mathrm{E}}}{\mathrm{X}_{\mathrm{bs}(\text { inicial })}-\mathrm{X}_{\mathrm{e}}}
$$

onde: RX é a razão de umidade (adimensional); $\mathrm{X}_{\mathrm{e}}$ é o teor de água de equilíbrio em base seca; $\mathrm{X}_{\mathrm{bs}}$ é o teor de água em base seca; $\mathrm{X}_{\mathrm{bs}(\text { inicial) }}$ é o teor de água inicial em base seca.

Com o cálculo da razão de teor de água, traçou-se a curva da cinética de secagem, representada pela razão do teor de água em função do tempo de secagem em minutos, aplicando os modelos matemáticos (Tabela 1) de Page, Henderson e Pabis e Logarítmico para ajustar os dados experimentais.

Tabela 1. Modelos matemáticos utilizados para descrever o processo de secagem.

\begin{tabular}{ll}
\hline Modelo & Equação \\
Page & $\mathrm{RX}=\exp \cdot\left(-\mathrm{k} . \mathrm{t}^{\mathrm{n}}\right)$ \\
Handerson \& Pabis & $\mathrm{RX}=\mathrm{a} \cdot \exp (-\mathrm{k} . \mathrm{t})$ \\
Logarítmico & $\mathrm{RX}=\mathrm{a} \cdot \exp -(\mathrm{kt})+\mathrm{c}$
\end{tabular}

Nota: RX é a razão de umidade (adimensional); k é a constante de secagem; "a", "n" e "b" são os parâmetros dos modelos; t é o tempo de secagem.

Fonte: Autores (2021).

Para análise dos ajustes dos modelos matemáticos aos dados experimentais, foi utilizado o programa computacional Statistica, versão sete, utilizando-se a análise de regressão não-linear, pelo método Quasi-Newton. Os modelos foram selecionados tomando-se como parâmetro a magnitude do coeficiente de determinação $\left(\mathrm{R}^{2}\right)$ e do desvio quadrático médio (DQM) (Equação 8).

$$
\mathrm{DQM}=\sqrt{\sum_{\mathrm{i}=1}^{\mathrm{n}} \frac{\left(\mathrm{RX}_{\exp }-\mathrm{RX}_{\mathrm{pred}}\right)^{2}}{\mathrm{~N}}}
$$

Em que: RXexp é a razão de teor de água obtida experimentalmente; RXpred é a razão de teor de água predita pelo modelo matemático; $\mathrm{N}$ é o número de observações ao longo da cinética de secagem. 
Os coeficientes de difusão efetivos foram determinados ajustando o modelo matemático da difusão líquida (Lei de Fick) com aproximação de três termos (Equação 9) aos dados experimentais da cinética de secagem das fatias do mesocarpo do maracujá, apenas para a melhor condição do planejamento fatorial da desidratação osmótica, considerando-se a distribuição da água inicial uniforme e a ausência de resistência térmica. Este modelo é a solução analítica para a segunda Lei de Fick, considerando-se a forma geométrica das fatias do mesocarpo do maracujá como aproximada a uma placa plana.

$$
\mathrm{RX}=\frac{8}{\pi^{2}} \sum_{\mathrm{n}=0}^{\infty} \frac{1}{(2 \mathrm{n}+1)^{2}} \exp \left[-(2 \mathrm{n}+1)^{2} \pi^{2} \mathrm{D}_{\mathrm{ef}} \frac{\mathrm{t}}{4 \mathrm{~L}^{2}}\right]
$$

onde: $\mathrm{D}_{e f}$ é o coeficiente de difusão efetivo $\left(\mathrm{m}^{2} \mathrm{~min}^{-1}\right)$; $\mathrm{n}$ é o número de termos da equação; L é a dimensão da casca; $\mathrm{t}$ é o tempo (min).

A relação entre a difusividade e as temperaturas de secagem foi descrita por meio da Equação do tipo Arrhenius (Equação 10).

$$
\operatorname{Def}=D_{0} \exp \left(-\frac{E_{a}}{R(T+273.15)}\right)
$$

onde: $\mathrm{D}_{0}$ é uma constante chamada de fator pré- exponencial $\left(\mathrm{m}^{2} \mathrm{~s}^{-1}\right)$; R é a constante universal dos gases ideais $(0,008314 \mathrm{~kJ}$ mol. $\left.\mathrm{K}^{-1}\right)$; T é a temperatura $\left({ }^{\circ} \mathrm{C}\right)$. Tal ajuste possibilita determinar a energia de ativação $\left(\mathrm{E}_{\mathrm{a}}\right), \mathrm{kJ} \mathrm{mol}^{-1}$.

As propriedades termodinâmicas de entalpia (Equação 11), entropia (Equação 12) e energia livre de Gibbs (Equação 13) relacionadas ao processo de secagem das fatias do mesocarpo do maracujá, na melhor condição do planejamento fatorial da desidratação osmótica, foram determinadas pelo método descrito pela constante universal dos gases (Silva, Rodovalho, Velasco, Silva \& Vale, 2016).

$$
\begin{aligned}
& \Delta H=E_{a}-R(T+273,15) \\
& \Delta S=R\left[\ln D_{0}-\ln \left(\frac{k_{b}}{h_{p}}\right)-\ln (T+273,15)\right]
\end{aligned}
$$

$$
\Delta \mathrm{G}=\Delta \mathrm{H}-(\mathrm{T}+273,15) \Delta \mathrm{S}
$$

onde: $\Delta \mathrm{H}$ é entalpia específica $\left(\mathrm{J} \mathrm{mol}^{-1}\right) ; \Delta \mathrm{S}$ é entropia específica $\left(\mathrm{J}\right.$ mol. $\left.\mathrm{K}^{-1}\right) ; \Delta \mathrm{G}$ é a energia livre de $\mathrm{Gibbs}\left(\mathrm{J}\right.$ mol$\left.{ }^{-1}\right) ; \mathrm{k}_{\mathrm{b}}$ é a constante de Boltzmann $\left(1,38 \times 10^{-23} \mathrm{~J} \mathrm{~K}^{-1}\right) ; \mathrm{h}_{\mathrm{p}}$ é a constante de Planck $\left(6,626 \times 10^{-34} \mathrm{~J} \mathrm{~s}^{-1}\right)$; T é a temperatura $\left({ }^{\circ} \mathrm{C}\right)$.

Através da linearização da Equação de Arrhenius, foi possível obter o coeficiente $\ln \mathrm{D}_{\mathrm{o}}$ da expressão de entropia ( $\Delta S$ ), em que foi utilizada a seguinte expressão (Equação 14): 


$$
\ln D_{\text {ef }}=\ln D_{0}-\frac{E a}{R} \cdot \frac{1}{(T+273,15)}
$$

\subsection{Caracterizações físicas e físico-químicas}

As fatias do mesocarpo do maracujá após processo de secagem da condição otimizada foi caracterizada quanto aos seguintes parâmetros: pH determinado por leitura direta pelo método potenciométrico; acidez total titulável (ATT), sólidos solúveis totais (SST), teor de água, sólidos totais e cinzas segundo a metodologia descrita por Brasil (2008). A atividade de água $\left(\mathrm{a}_{\mathrm{w}}\right.$ ) foi determinada diretamente em equipamento Aqualab 3TE, da Decagon Devices a $25{ }^{\circ} \mathrm{C}$; os açúcares redutores, açúcares não redutores e açúcares totais pelo método de Lane e Eynon (1934). Os parâmetros de cor foram determinados utilizando-se o espectrofotômetro Mini Scan Hunter Lab XE Plus (Reston, VA, EUA), no sistema de cor Cielab. O qual permitiu a obtenção dos parâmetros: $L^{*}$ (luminosidade); a* (transição da cor verde $\left(-a^{*}\right)$ para o vermelho $\left(+a^{*}\right)$ ); e b* (transição da cor azul (-b*) para a cor amarela $\left.\left(+b^{*}\right)\right)$.

\subsection{Análise estatística}

As análises estatísticas foram realizadas para os dados experimentais em triplicata e os resultados foram submetidos à análise de variância de fator único (ANOVA) de $5 \%$ de probabilidade e as respostas qualitativas significativas foram submetidas ao teste de Tukey adotando-se o mesmo nível de 5\% de significância. Para o desenvolvimento das análises estatísticas o software Assistat versão 7.0 foi utilizado.

\subsection{Resultados da pesquisa}

Na Tabela 2 estão apresentados os valores das variáveis dependentes, encontrados nos diferentes experimentos do planejamento experimental fatorial, em função das variáveis independentes.

Tabela 2. Respostas dos experimentos da desidratação osmótica em função da concentração de sacarose e temperatura.

\begin{tabular}{|c|c|c|c|c|c|}
\hline \multirow[b]{2}{*}{ Ensaios } & \multicolumn{2}{|c|}{$\begin{array}{c}\text { Variáveis } \\
\text { independentes }\end{array}$} & \multicolumn{3}{|c|}{ Respostas } \\
\hline & $\begin{array}{c}\mathrm{CS} \\
\left({ }^{\circ} \mathrm{Brix}\right)\end{array}$ & $\mathrm{T}\left({ }^{\circ} \mathrm{C}\right)$ & $\begin{array}{l}\mathrm{PA} \\
(\%)\end{array}$ & $\begin{array}{l}\mathrm{PM} \\
(\%)\end{array}$ & $\begin{array}{l}\text { GS } \\
(\%)\end{array}$ \\
\hline 1 & $-1(40)$ & $-1(40)$ & 26,69 & 25,27 & 1,42 \\
\hline 2 & $+1(60)$ & $-1(40)$ & 50,39 & 37,97 & 12,42 \\
\hline 3 & $-1(40)$ & $+1(60)$ & 49,42 & 39,42 & 10,00 \\
\hline 4 & $+1(60)$ & $+1(60)$ & 57,23 & 42,35 & 14,88 \\
\hline 5 & $0(50)$ & $0(50)$ & 48,66 & 39,97 & 8,69 \\
\hline 6 & $0(50)$ & $0(50)$ & 48,57 & 39,37 & 9,20 \\
\hline 7 & $0(50)$ & $0(50)$ & 46,60 & 38,60 & 8,00 \\
\hline
\end{tabular}

Nota: CS é a concentração de sacarose; T é a temperatura; PA é a perda de água; PM é a perda de massa; GS é o ganho de sólidos.

Fonte: Autores (2021). 
Constata-se que os valores de perda de água (PA) variaram entre 26,69\% e 57,23\%, sendo que o maior valor foi encontrado no ensaio 4, para as condições $(+1)$ de concentração de sacarose e temperatura. O menor valor foi do ensaio 1 , onde utilizou-se os menores valores das variáveis independentes, resultando na menor perda de massa (PM), os quais apresentaram valores entre $25,27 \%$ e $42,35 \%$. O mesmo comportamento para perda de água e perda de massa também foi verificado por Silva Júnior et al. (2020), na otimização da osmodesidratação das cascas de banana.

Com relação ao ganho de sólidos (GS), este apresentou maiores valores nos ensaios que apontam as maiores concentrações de sacarose, ensaios 2 e 4, porém, salienta-se que houve alta concentração deste fator no ensaio 3, mesmo com a concentração de sacarose (-1), logo isto veio a influenciar consideravelmente no processo. Paglarini et al. (2015) em seus estudos com desidratação osmótica, os ensaios com menor concentração de sacarose e maior temperatura mostrou-se como o de maior porcentagem de ganho de sólidos, assim como observado no presente estudo.

A Tabela 3 demonstra a análise de variância (ANOVA) do modelo ajustado para as variáveis de resposta da desidratação osmótica. Verifica-se a significância da regressão e a da falta de ajuste a 95\% de confiança, pelo teste F.

Tabela 3. Análise de variância (ANOVA) dos modelos ajustados para as variáveis de resposta.

\begin{tabular}{|c|c|c|c|c|}
\hline $\begin{array}{l}\text { Fonte de } \\
\text { variação }\end{array}$ & $\mathrm{F}_{\mathrm{c}}$ & $\mathrm{F}_{\mathrm{t}}$ & $\mathrm{F}_{\mathrm{c}} / \mathrm{F}_{\mathrm{t}}$ & $\mathrm{R}^{2}$ \\
\hline \multicolumn{5}{|c|}{ Perda de água } \\
\hline Regressão & 54,95 & 9,28 & 5,92 & 90,94 \\
\hline $\begin{array}{c}\text { Falta de } \\
\text { ajuste }\end{array}$ & 5,11 & 18,51 & 0,27 & \\
\hline \multicolumn{5}{|c|}{ Perda de massa } \\
\hline Regressão & 10,04 & 9,28 & 1,08 & 98,21 \\
\hline $\begin{array}{c}\text { Falta de } \\
\text { ajuste }\end{array}$ & 34,05 & 18,51 & 1,83 & \\
\hline \multicolumn{5}{|c|}{ Ganho de sólidos } \\
\hline Regressão & 39,33 & 9,28 & 4,23 & 97,52 \\
\hline $\begin{array}{c}\text { Falta de } \\
\text { ajuste }\end{array}$ & 5,21 & 18,51 & 0,28 & \\
\hline
\end{tabular}

Nota: Fc é o F calculado; Ft é o F tabelado.

Fonte: Autores (2021).

Observa-se que para a regressão em todas as variáveis analisadas, o valor de $\mathrm{F}_{\text {calculado }}$ foi maior que o valor de $\mathrm{F}_{\text {tabelado, }}$, logo o modelo linear foi estatisticamente significativo. Para a perda de água e ganho de sólidos o modelo foi considerado preditivo, visto que para um modelo ser estatisticamente preditivo, a razão $F_{\text {calculado }} / F_{\text {tabelado }}>4$. A falta de ajuste foi significativa apenas para a perda de massa, ou seja, houve uma pequena falta de ajuste dos dados ao modelo citado.

A Figura 1 mostra o diagrama de Pareto das variáveis dependentes durante o processo de desidratação osmótica, representando os efeitos dos parâmetros de regressão e suas respectivas significâncias. A magnitude dos efeitos é representada pelas barras horizontais, enquanto que a linha perpendicular representa a magnitude dos efeitos com significado estatístico para $P=0,05$. 
Research, Society and Development, v. 10, n. 13, e122101320771, 2021

(CC BY 4.0) | ISSN 2525-3409 | DOI: http://dx.doi.org/10.33448/rsd-v10i13.20771

Figura 1. Diagramas de Pareto para a perda de água, perda de massa e ganho de sólidos.
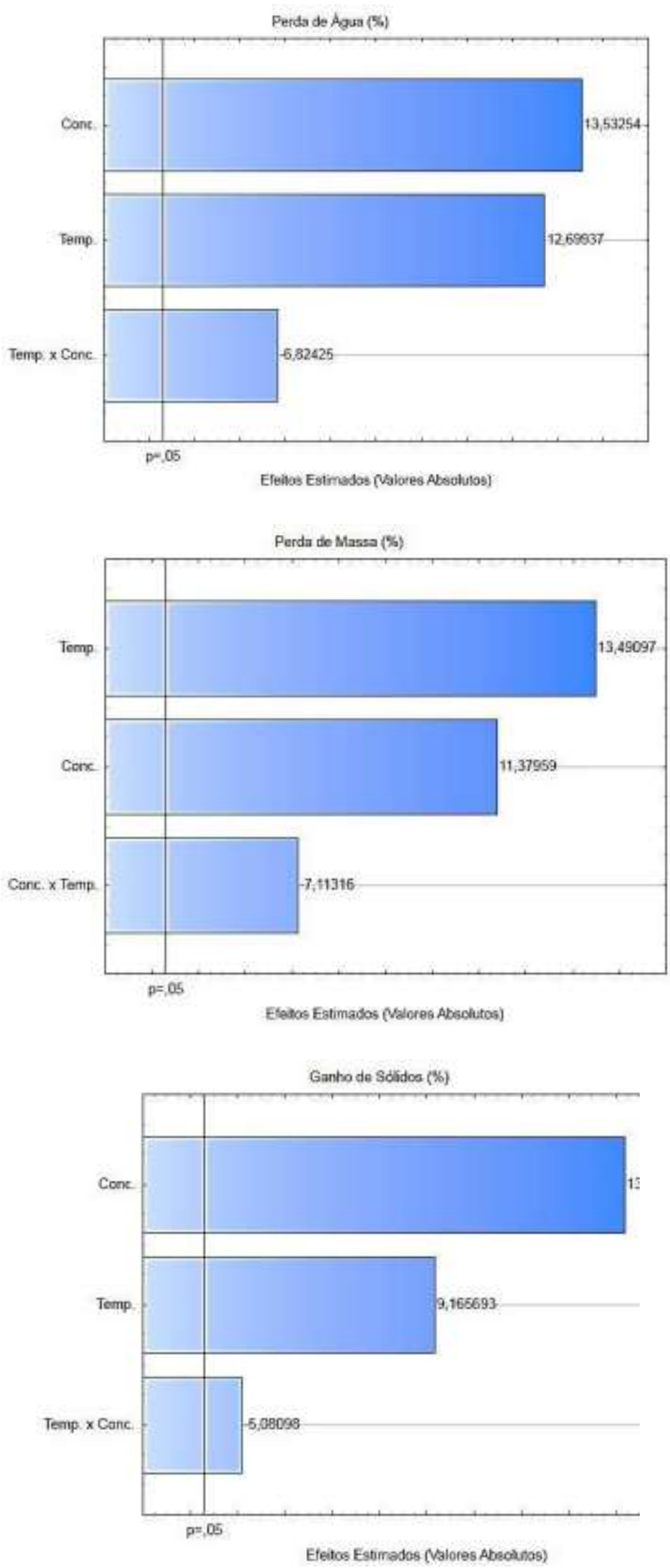

Fonte: Autores (2021). 
Para todas as variáveis de resposta observa- se que todos os parâmetros ultrapassaram a linha transversal, tendo efeito positivo a $95 \%$ de confiança, considerando também a interação entre elas. Constata-se que para a perda de água e para o ganho de sólidos a concentração de sacarose foi o fator que mais influenciou na resposta estudada, apresentando efeito positivo. Quanto maior a concentração de sacarose na solução osmótica, maior o ganho de sólidos das amostras. No entanto, para a perda de massa a temperatura teve maior influência no processo.

A Figura 2 apresenta as superfícies de respostas para as variáveis de perda de água, perda de massa e ganho de sólidos das fatias do mesocarpo do maracujá.

Figura 2. Superfície de resposta da perda de água, perda de massa e ganho de sólidos das fatias do mesocarpo de maracujá, relacionando a concentração de sacarose e temperatura da solução osmótica.

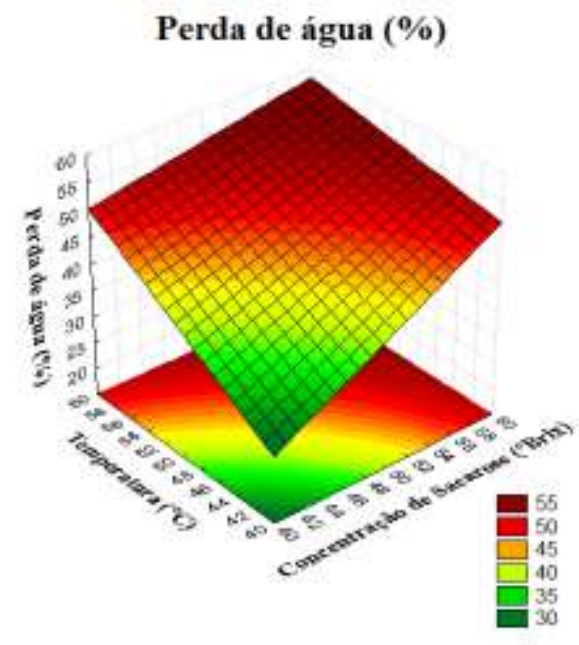

Perda de massa $(\%)$

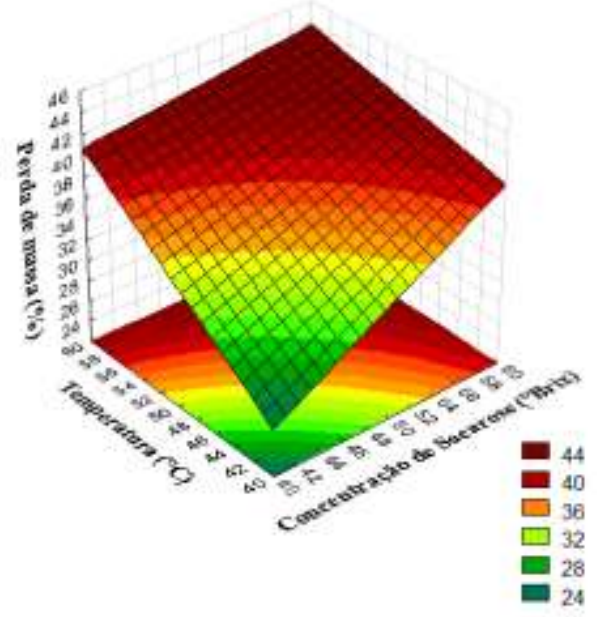




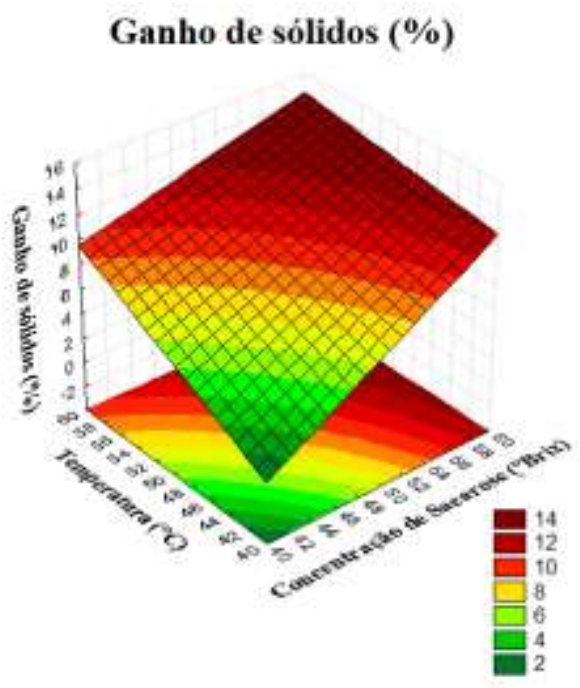

Fonte: Autores (2021).

O aumento dos fatores favoreceu a perda de massa e a perda de água e ganho de sólidos. A geometria de placas planas das fatias contribuiu para facilitar a incorporação de sólidos no início do processo, devido à maior área de contato da fruta com a solução desidratante. Durante os ensaios realizados pode-se perceber que a partir de 60 minutos do processo de desidratação osmótica ocorreu grande diminuição do teor de água, como também, uma incorporação de solutos. Levando em consideração os efeitos observados durante a desidratação osmótica, e tendo em vista que o objetivo principal é aumentar sua vida de prateleira, foi definido que o ensaio $4\left(60^{\circ}\right.$ Brix e $\left.60^{\circ} \mathrm{C}\right)$ seria a melhor condição do processo, pois apresentou maior perda de água, perda de massa e ganho de sólidos.

As fatias do mesocarpo do maracujá osmodesidratada a $60{ }^{\circ}$ Brix e $60{ }^{\circ} \mathrm{C}$ (ensaio 4), foram submetidas a cinética de secagem nas temperaturas de 50, 60 e $70{ }^{\circ} \mathrm{C}$. A Tabela 4 apresenta os valores obtidos pelo ajuste dos modelos matemáticos aos dados experimentais da cinética de secagem.

Tabela 4 - Parâmetros obtidos pelo ajuste dos modelos matemáticos aos dados experimentais da cinética de secagem das fatias do mesocarpo de maracujá osmodesidratada a $60{ }^{\circ}$ Brix e $60^{\circ} \mathrm{C}$.

\begin{tabular}{|c|c|c|c|c|c|c|}
\hline \multirow{2}{*}{ Modelo } & \multirow{2}{*}{$\begin{array}{c}\mathrm{T} \\
\left({ }^{\circ} \mathrm{C}\right)\end{array}$} & \multicolumn{3}{|c|}{ Parâmetros } & \multirow{2}{*}{$\mathrm{R}^{2}$} & \multirow{2}{*}{ DQM } \\
\hline & & $\mathbf{k}$ & & & & \\
\hline \multirow{3}{*}{ Page } & 50 & 0,026 & \multicolumn{2}{|c|}{0,750} & 0,997 & 0,0676 \\
\hline & 60 & 0,028 & \multicolumn{2}{|c|}{0,789} & 0,995 & 0,0679 \\
\hline & 70 & 0,029 & \multicolumn{2}{|c|}{0,878} & 0,997 & 0,0594 \\
\hline \multirow{4}{*}{$\begin{array}{l}\text { Handerson } \\
\text { e Pabis }\end{array}$} & $\begin{array}{c}\mathrm{T} \\
\left({ }^{\circ} \mathrm{C}\right) \\
\end{array}$ & $\mathrm{a}$ & \multicolumn{2}{|c|}{$\mathrm{k}$} & $\mathrm{R}^{2}$ & DQM \\
\hline & 50 & 0,929 & \multicolumn{2}{|c|}{0,007} & 0,992 & 0,1058 \\
\hline & 60 & 0,960 & \multicolumn{2}{|c|}{0,010} & 0,992 & 0,0978 \\
\hline & 70 & 0,985 & \multicolumn{2}{|c|}{0,017} & 0,996 & 0,0703 \\
\hline \multirow{4}{*}{ Logarítmico } & $\begin{array}{c}\mathrm{T} \\
\left({ }^{\circ} \mathrm{C}\right) \\
\end{array}$ & A & $\mathrm{k}$ & $\mathrm{c}$ & $\mathrm{R}^{2}$ & DQM \\
\hline & 50 & 0,915 & 0,007 & 0,031 & 0,996 & $\begin{array}{c}0,0620 \mathrm{x} \\
10^{-5}\end{array}$ \\
\hline & 60 & 0,945 & 0,011 & 0,030 & 0,996 & $\begin{array}{c}0,0070 \times \\
10^{-5}\end{array}$ \\
\hline & 70 & 0,971 & 0,018 & 0,020 & 0,998 & $\begin{array}{c}0,0003 \times \\
10^{-5} \\
\end{array}$ \\
\hline
\end{tabular}


Fonte: Autores (2021).

Todos os modelos ajustados apresentaram coeficiente de determinação $\left(\mathrm{R}^{2}\right)$ maior que 0,99 para as três temperaturas aplicadas. A qualidade do ajuste dos modelos aos dados experimentais pode ser avaliada com diferentes índices estatísticos. Contudo, de acordo com Kucuk, Midilli, Kilic e Dincer (2014) e Ferreira et al. (2020), o melhor modelo para descrever a curva de secagem do produto é a que apresenta os maiores valores de coeficiente de determinação $\left(\mathrm{R}^{2}\right)$ e os menores valores de desvio quadrado médio. Sendo assim, ao considerar os valores do desvio quadrado médio (DQM), o modelo Logarítmico apresentou os mais baixos quando comparados aos valores obtidos nos modelos de Page e de Handerson e Pabis, sendo considerado assim o melhor modelo de ajuste aos dados experimentais.

Silva et al. (2019) ao realizar a cinética de secagem do mesocarpo do maracujá sem pré-tratamento osmótico nas temperaturas de 70 e $80{ }^{\circ} \mathrm{C}$, obtiveram que o modelo de Page foi o que melhor se ajustou aos dados experimentais.

Na Figura 3 observa-se as constantes de secagem $(\mathrm{k})$ dos modelos matemáticos ajustados aos dados experimentais (Page, Handerson e Pabis e Logarítmico), no qual o valor aumentou de acordo com o aumento da temperatura do ar de secagem. Segundo Lisboa et al. (2019) este é um resultado esperado, uma vez que a constante de secagem representa o efeito das condições de secagem externas e tende a ter este perfil. Os parâmetros "a" e " $n$ " dos modelos ajustados também apresentaram comportamento linear similar ao observado para constante de secagem (k).

Figura 3. Constante de secagem (k) dos modelos de Page, Handerson e Pabis e Logarítmico.

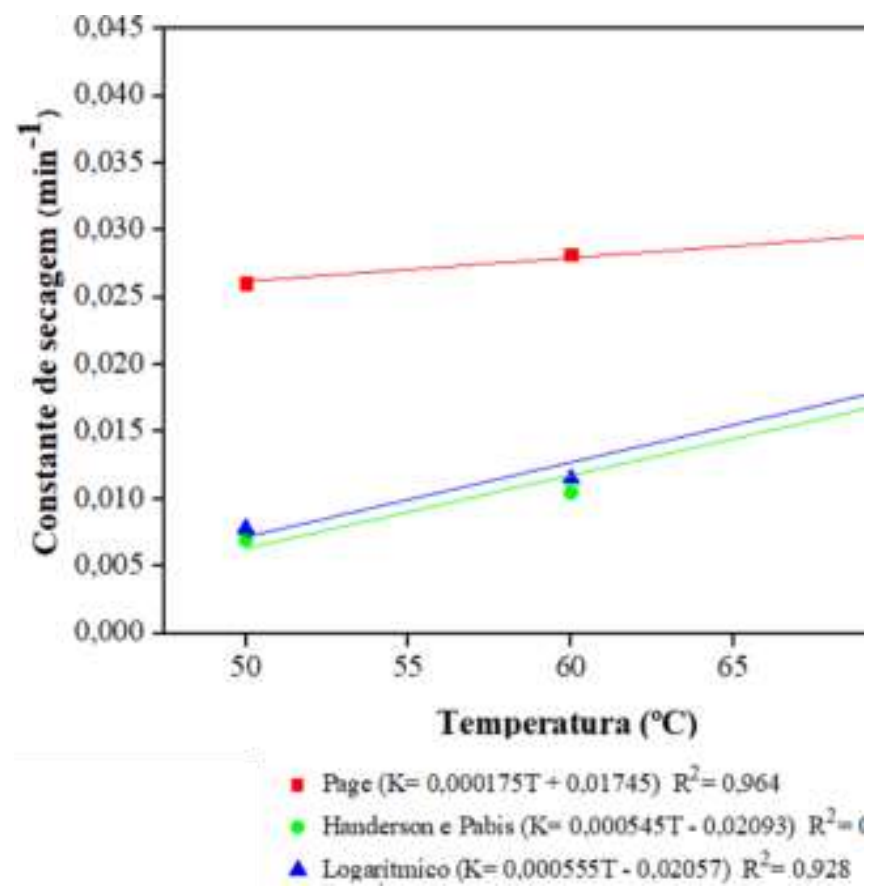

Fonte: Autores (2021).

Observa-se que as fatias do mesocarpo do maracujá submetidas à menor temperatura de secagem, $50{ }^{\circ} \mathrm{C}$ necessitou mais tempo até atingir o teor de água final. Com o aumento da temperatura, a transferência de massa foi favorecida, diminuindo a umidade de equilíbrio dinâmico e o tempo de secagem.

Na Tabela 5 estão apresentados os valores obtidos pelo ajuste do modelo difusional de Fick e as propriedades termodinâmicas da cinética de secagem das fatias do mesocarpo de maracujá osmodesidratada a $60{ }^{\circ} \mathrm{Brix}$ e $60{ }^{\circ} \mathrm{C}$. 
Tabela 5. Valores da difusividade efetiva, coeficientes de determinação $\left(\mathrm{R}^{2}\right)$ e propriedades termodinâmicas da cinética de secagem das fatias do mesocarpo de maracujá osmodesidratada a $60{ }^{\circ}$ Brix e $60^{\circ} \mathrm{C}$.

\begin{tabular}{cccccc}
\hline $\mathrm{T}\left({ }^{\circ} \mathrm{C}\right)$ & $\mathrm{D}_{\mathrm{ef}}$ & $\mathrm{R}^{2}$ & $\Delta \mathrm{H}$ & $\Delta \mathrm{S}$ & $\Delta \mathrm{G}$ \\
\hline 50 & 1,00 & 0,9997 & 40,62 & $-299,84$ & 137,19 \\
60 & 3,50 & 0,9996 & 40,53 & $-299,59$ & 140,18 \\
70 & 3,95 & 0,9995 & 40,45 & $-299,34$ & 143,17 \\
\hline
\end{tabular}

Nota: Def x $10^{-5}\left(\mathrm{~m}^{2} \mathrm{~min}^{-1}\right)$; é a entalpia $\left(\mathrm{Kj} \mathrm{mol}^{-1}\right)$; é a entropia $\left(\mathrm{J}\right.$ mol. $\left.\mathrm{K}^{-1}\right)$; é a energia livre de Gibbis $\left(\mathrm{kJ} \mathrm{mol}^{-1}\right)$.

Fonte: Autores (2021)

Os valores de difusividade obtidos variaram entre 1,00 a 3,95 x $10^{-5} \mathrm{~m}^{2} \mathrm{~min}^{-1}$ quando atemperatura de secagem variou de 50 a $70{ }^{\circ} \mathrm{C}$. Ferreira et al. (2020) obtiveram valores de difusividade variando de 1,268 a $1,976 \times 10^{-9} \mathrm{~m} \mathrm{~s}^{-1}$ para cubos de mamão osmodesidratado a $50{ }^{\circ}$ Brix e $60{ }^{\circ} \mathrm{C}$ quando a temperatura de secagem variou de 50 a $70{ }^{\circ} \mathrm{C}$. Aires et al. (2016) observaram valores de difusividade de $3,19 \times 10^{-10} \mathrm{~m} \mathrm{~s}^{-1}$ para cubos de maçã desidratados a $40{ }^{\circ}$ Brix e $40{ }^{\circ} \mathrm{C}$. Para Almeida et al. (2020), na medida em que se aumenta o gradiente de temperatura, maior é o valor obtido para este parâmetro, pois em temperaturas inferiores como a de $50{ }^{\circ} \mathrm{C}$ as moléculas de água sofrem maior resistência ao sair do corpo devido a sua composição e estrutura física, fazendo com que a água fique mais ligada às moléculas que constituem a massa seca.

Por meio do ajuste da Equação de Arrhenius, o processo de secagem apresentou uma energia de ativação (Ea) de 43,31 $\mathrm{kJ} \mathrm{mol}^{-1}$, no qual encontra-se dentro da faixa apresentada por Zogzas, Mauroulis e Marinos-Kouris (1996), onde afirma que a energia de ativação varia para produtos agrícolas entre 12,7-110 kJ mol-1 . Gomes, Osvaldo, Sousa, Oliveira e Araújo Neto (2018) em seus estudos de secagem com a massa triturada de jambu obtiveram uma energia de ativação que variou de 16,61 a 16,97 kJ mol${ }^{-1}$. Corrêa, Resende, Martinazzo, Goneli e Botelho (2007) e Araújo, Goneli, Corrêa, Hartman Filho e Martins (2017), relatam que a energia de ativação indica a facilidade com que as moléculas de água superam a barreira de energia durante a migração no interior do produto, uma vez que nos processos de secagem quanto menor a energia de ativação, maior será a difusividade da água no produto.

A entalpia apresentou valores positivos que variaram de 40,62 a 40,45 $\mathrm{kJ} \mathrm{mol}^{-1}$ reduzindo conforme se teve aumento da temperatura de secagem. Segundo Carvalho, Bueno, Oliveira e Resende (2018) isso indica que menor quantidade de energia é requerida para que a secagem ocorra em temperaturas mais elevadas. Houve redução nos valores da entropia quando se teve aumento da temperatura de secagem, vaiando de $-299,34\left(70{ }^{\circ} \mathrm{C}\right)$ a $-299,89 \mathrm{~J}$ mol. $\mathrm{K}^{-1}\left(50^{\circ} \mathrm{C}\right)$. Segundo Almeida et al. (2020) isso acontece devido à redução no teor de umidade ao longo do processo de secagem, o que dificulta o movimento das moléculas de água. Os valores da energia livre de Gibbs aumentaram com o aumento da temperatura de secagem variando de 137,19 a 143,17 kJ mol ${ }^{-1}$ indicando que o processo de secagem ocorreu de forma não espontânea.

Na Tabela 6 estão apresentados os valores experimentais encontrados na caracterização físico-química das fatias osmodesidratadas e secas do mesocarpo do maracujá. 
Tabela 6. Caracterização físico-química das fatias osmodesidratadas $\left(60{ }^{\circ}\right.$ Brix e $\left.60{ }^{\circ} \mathrm{C}\right)$ e secas do mesocarpo do maracujá secas em diferentes temperaturas.

\begin{tabular}{|c|c|c|c|}
\hline \multirow{2}{*}{ Parâmetros } & \multicolumn{3}{|c|}{ Temperaturas $\left({ }^{\circ} \mathrm{C}\right)$} \\
\hline & $50^{\circ} \mathrm{C}$ & $60^{\circ} \mathrm{C}$ & $70^{\circ} \mathrm{C}$ \\
\hline $\mathrm{pH}$ & $4,00^{\mathrm{b}}$ & $4,18^{\mathrm{a}}$ & $3,94^{\mathrm{c}}$ \\
\hline $\begin{array}{l}\text { Acidez total titulável (\% } \\
\text { ácido cítrico) }\end{array}$ & $0,720^{\mathrm{a}}$ & $0,719^{\mathrm{a}}$ & $0,770^{\mathrm{a}}$ \\
\hline $\begin{array}{l}\text { Sólidos solúveis totais } \\
\left.\text { ( }{ }^{\circ} \text { Brix }\right)\end{array}$ & $64,50^{c}$ & $66,60^{\mathrm{b}}$ & $68,40^{\mathrm{a}}$ \\
\hline Teor de água (\%b.u) & $16,76^{\mathrm{a}}$ & $11,89^{\mathrm{b}}$ & $8,82^{\mathrm{c}}$ \\
\hline Sólidos totais (\%) & $83,24^{\mathrm{c}}$ & $88,11^{\mathrm{b}}$ & $91,51^{\mathrm{a}}$ \\
\hline Atividade de água $\left(\mathrm{a}_{\mathrm{w}}\right)$ & $0,688^{\mathrm{a}}$ & $0,511^{\mathrm{b}}$ & $0,485^{\mathrm{c}}$ \\
\hline Cinzas $(\%)$ & $0,68^{a}$ & $0,66^{\mathrm{a}}$ & $0,67^{\mathrm{a}}$ \\
\hline $\begin{array}{l}\text { Açúcares redutores } \\
\qquad(\mathrm{g} / 100 \mathrm{~g})\end{array}$ & $5,42^{\mathrm{c}}$ & $7,54^{\mathrm{b}}$ & $12,41^{\mathrm{a}}$ \\
\hline $\begin{array}{l}\text { Açucares não redutores } \\
\qquad(\mathrm{g} / 100 \mathrm{~g})\end{array}$ & $77,59^{\mathrm{a}}$ & $78,62^{\mathrm{a}}$ & $77,42^{\mathrm{a}}$ \\
\hline Açucares totais $(\mathrm{g} / 100 \mathrm{~g})$ & $83,01^{\mathrm{c}}$ & $86,17^{\mathrm{b}}$ & $89,83^{\mathrm{a}}$ \\
\hline Luminosidade $\left(\mathrm{L}^{*}\right)$ & $76,76^{\mathrm{a}}$ & $75,89^{\mathrm{b}}$ & $73,32^{\mathrm{c}}$ \\
\hline $\begin{array}{l}\text { Intensidade de vermelho } \\
\qquad\left(+\mathrm{a}^{*}\right)\end{array}$ & $1,06^{\mathrm{c}}$ & $2,83^{\mathrm{b}}$ & $4,58^{\mathrm{a}}$ \\
\hline $\begin{array}{l}\text { Intensidade de amarelo } \\
\qquad\left(+\mathrm{b}^{*}\right)\end{array}$ & $19,23^{\mathrm{c}}$ & $20,23^{\mathrm{b}}$ & $27,29^{\mathrm{a}}$ \\
\hline
\end{tabular}

Nota: As médias seguidas pela mesma letra na mesma linha não diferem estatisticamente de acordo com o teste de Tukey a 5\% de probabilidade Fonte: Autores (2021).

Os valores médios de pH variaram entre 3,94 a 4,18, os valores obtidos foram inferiores a 4,5 delimitando o crescimento de microrganismos deteriorantes. Valores superiores foram observados por Leite, Paglarini, Pinto, Silva e Porto (2011) para o albedo da melancia que apresentou variação de 5,03 a 5,15. Com relação ao parâmetro de acidez total titulável houve uma variação de 0,719 a 0,770\% ácido cítrico, não apresentando diferença estatística significativa. Valores inferiores de acidez (0,29\% de ácido cítrico) foram observados por Aragão, Loss, Sousa e Guedes (2017), ao analisarem mangas desidratadas osmoticamente.

A concentração de sólidos solúveis totais foi maior nas fatias secas a $70{ }^{\circ} \mathrm{C}$ que apresentou $68,40{ }^{\circ}$ Brix. Observou-se aumento nas concentrações de sólidos solúveis totais quando se teve aumento da temperatura de secagem, consequentemente, para as fatias secas a $50^{\circ} \mathrm{C}$ ocorreu a menor concentração de sólidos solúveis totais $\left(64,50{ }^{\circ}\right.$ Brix $)$, devido a menor incorporação da sacarose. Souza Neto et al. (2005) ao submeterem a manga a um tratamento osmótico sob pressão atmosférica (xarope a $55^{\circ}$ Brix por 4 horas) e secagem em estufa (por 16 horas), obtiveram $75^{\circ}$ Brix no produto final.

Os parâmetros de teor de água e atividade de de secagem e estes diferiram estatisticamente entre si. Para temperatura de $60{ }^{\circ} \mathrm{C}$ as fatias apresentaram 11,89\% de água, valor este próximo ao observado por Monteiro et al. (2020), ao secarem em Air fryer a $90{ }^{\circ} \mathrm{C}$ cubos de berinjela desidratados osmoticamente $(14,18 \%)$. A quantidade de sólidos totais foi maior na temperatura de $70{ }^{\circ} \mathrm{C}$, o que já era esperado, devido ao aumento da evaporação de água livre com o aquecimento e consequentemente maior concentração dos nutrientes. 
O teor de cinzas apresentou valores que variaram de 0,66 a $0,68 \%$, não diferindo estatisticamente entre as temperaturas de secagem aplicadas. Valores próximos ao do presente estudo foram relatados por Silva, Riguedo, Loss, Guedes e Carvalho (2017), que obtiveram 0,69\% em polpas de pequi desidratadas em uma solução de sacarose a $70 \%$ por 6 horas.

Os açúcares redutores, não redutores e totais das amostras secas aumentaram com aumento da temperatura de secagem. Pois com a ascendência da temperatura a viscosidade da solução de sacarose aumenta e isto causa penetração desta nas fatias, elevando assim os teores de açúcares redutores e totais. Para as três temperaturas de secagem observou-se diferença estatística para os parâmetros água $\left(\mathrm{a}_{\mathrm{w}}\right)$ diminuíram com aumento da temperatura de açúcares redutores e totais. No entanto, os teores de açúcares não redutores não diferiram estatisticamente entre as temperaturas de secagem. Valores inferiores ao do presente estudo foram observados por Castro et al. (2016) que obtiveram 34,83 g/100g de açúcares redutores e 51,70 g/100g de açúcares totais para goiabas osmodesidratadas $\left(50{ }^{\circ} \mathrm{Brix} / 50{ }^{\circ} \mathrm{C}\right)$ e secas a $70{ }^{\circ} \mathrm{C}$.

Analisando a cor das fatias do mesocarpo do maracujá, a luminosidade permaneceu maior na menor temperatura e (+a*) e $\left(+b^{*}\right)$ se intensificaram com a maior intensidade do calor nas amostras, onde a coloração vermelha e amarela foi acentuada com a incorporação de $30 \%$ da polpa as amostras.

\section{Conclusão}

A melhor condição de processo durante a desidratação osmótica foi a do experimento $4\left(60{ }^{\circ} \mathrm{Brix} / 60{ }^{\circ} \mathrm{C}\right)$, com maiores porcentagens para perda de água, perda de massa e ganho de sólidos. O modelo matemático Logarítmico foi o que melhor se ajustou aos dados experimentais da cinética de secagem, apresentando coeficiente de determinação $\left(\mathrm{R}^{2}\right)$ próximos a 1 e baixos valores do desvio quadrático médio (DQM). A difusividade efetiva foi maior para as fatias secas a $70{ }^{\circ} \mathrm{C}, \mathrm{o}$ processo de secagem apresentou uma energia de ativação de $43,31 \mathrm{~kJ} \mathrm{~mol}^{-1}$. As propriedades termodinâmicas indicaram que o processo de secagem foi endorgênico e não espontâneo.

Mesmo ocorrendo variações nos parâmetros físicos e físico-químicos após a desidratação osmótica seguida de secagem convectiva complementar, torna-se viável o aproveitamento desse resíduo como forma alternativa na alimentação, além de minimizar os impactos ambientais causados quando lançados no meio ambiente.

\section{Referências}

Aires, J. E. F., da Silva, W. P., Aires, K. L. C. D. A. F., da Silva Júnior, A. F., \& Silva, C. M. D. P. D. S. E. (2017). Description of osmotic dehydration of apple using two-dimensional diffusion models considering shrinkage and variations in process parameters. Drying Technology, 35(7), 815-826.

Almeida, R. L. J., Santos, N. C., Pereira, T. P., Queiroga, A. P. R., Silva, V. M. A., Ribeiro, V. H. A., \& Borges, E. M. E. S. (2020). Cinética de secagem do feijão azuki: modelagem matemática e propriedades termodinâmicas. Research, Society and Development, 9(3), e27932316.

Aragão, P. P., Loss, R. A., Sousa, S., \& Guedes, S. F. (2017). Avaliação do potencial de utilização de pré-tratamento osmótico na produção de manga desidratada. Revista Destaques Acadêmicos, 9(4).

Araujo, W. D., Goneli, A. L. D., Corrêa, P. C., Hartmann, C. P., \& Martins, E. A. S. (2017). Modelagem matemática da secagem dos frutos de amendoim em camada delgada1. Revista Ciência Agronômica, 48, 448-457.

Brasil. (2008). Instituto Adolfo Lutz. Métodos físico-químicos para análise de alimentos. ANVISA.

Carvalho, J. M. G., Bueno, S. G. S., de Oliveira, D. E. C., \& Resende, O. (2018). Modelagem matemática e propriedades termodinâmicas da secagem do grão de girassol. Global Science and Technology, 11(2).

Castro, D. S., Aires, J. E. F., Aires, K. L. C., da Silva, A. F., da Silva, W. P., \& Gomes, J. P. (2016). Physical and chemical changes in guava raisin ('Psidum guajava'1.) produced by osmotic dehydration and drying convective. Australian Journal of Crop Science, 10(10), $1449-1454$.

Cavalcante, N. R., Viana, A. P., Almeida Filho, J. E., Pereira, M. G., Ambrósio, M., Santos, E. A., \& Sousa, C. M. B. (2019). Novel selection strategy for halfsib families of sour passion fruit Passiflora edulis (Passifloraceae) under recurrent selection. Genetics and Molecular Research, 18(2), gmr18305-gmr18305.

Corrêa, P. C., Resende, O., Martinazzo, A. P., Goneli, A. L., \& Botelho, F. M. (2007). Modelagem matemática para a descrição do processo de secagem do feijão (Phaseolus vulgaris L.) em camadas delgadas. Engenharia Agrícola, 27, 501-510. 
Costa, C. F., Fusieger, A., Andretta, M., Camargo, A. C., Carvalho, A. F., Menezes, D. R., \& Nero, L. A. (2020). Potential use of passion fruit (Passiflora cincinnata) as a biopreservative in the production of coalho cheese, a traditional Brazilian cheese. Journal of dairy science, $103(4), 3082-3087$.

Ferreira, J. P. D. L., Castro, D. S. D., Moreira, I. D. S., Silva, W. P. D., de Figueirêdo, R. M., \& Queiroz, A. J. D. M. (2020). Convective drying kinetics of osmotically pretreated papaya cubes. Revista Brasileira de Engenharia Agrícola e Ambiental, 24, 200-208.

Gomes, F. P., Osvaldo, R., Sousa, E. P., de Oliveira, D. E., \& Araújo, F. R. D. (2018). Drying kinetics of crushed mass of 'jambu': Effective diffusivity and activation energy. Revista Brasileira de Engenharia Agrícola e Ambiental, 22, 499-505.

IBGE. (2019). Instituto Brasileiro de Geografia e Estatística. https://sidra. ibge.gov.br/tabela/5457/.

Kucuk, H., Midilli, A., Kilic, A., \& Dincer, I. (2014). A review on thin-layer drying-curve equations. Drying Technology, 32(7), 757-773.

Kulkarni, S. G., \& Vijayanand, P. (2010). Effect of extraction conditions on the quality characteristics of pectin from passion fruit peel (Passiflora edulis f. flavicarpa L.). LWT-Food Science and Technology, 43(7), 1026-1031.

Lane, J. H., \& Eynon, L. (1934). Determination of reducing sugars by Fehling's solution with methylene blue indicator. N. Rodger.

Leite, A. L., Paglarini, C., Pinto, E., Silva, F., \& Porto, A. (2011). Influência da desidratação osmótica seguida de secagem nas características físico, químicas e sensoriais do albedo de melancia. Enciclopédia Biosfera, 7(13).

Lima, L. K. S., Jesus, O. N., Soares, T. L., dos Santos, I. S., de Oliveira, E. J., \& Coelho Filho, M. A. (2020). Growth, physiological, anatomical and nutritional responses of two phenotypically distinct passion fruit species (Passiflora L.) and their hybrid under saline conditions. Scientia Horticulturae, 263 , 109037.

Lisboa, H. M., Araujo, H., Paiva, G., Oriente, S., Pasquali, M., Duarte, M. E., \& Mata, M. E. C. (2019). Determination of characteristic properties of mulatto beans (Phaseolus vulgaris L.) during convective drying. Journal of Agriculture and Food Research, 1, 100003.

Monteiro, S. S., Monteiro, S. S., Santos, N. C., Barros, S. L., Cruz, O. N., Martins, L. P., \& Gomes, J. P. (2020). Aplicação de processos combinados osmótico e secagem em air fryer em berinjelas condimentadas com hibisco. Research, Society and Development, 9(3), e07932267-e07932267.

Nascimento, T. A., Calado, V., \& Carvalho, C. W. P. (2012). Development and characterization of flexible film based on starch and passion fruit mesocarp flour with nanoparticles. Food Research International, 49(1), 588-595.

Paglarini, C. S., Silva, F. S., Porto, A. G., Zela, S. P., Leite, A. L. M. P., Furtado, G. F. (2015). Efeito das condições de desidratação osmótica na qualidade de passas de araçá-pêra. Revista Brasileira de Tecnologia Agroindustrial, 9(2), 1945-1961.

Panarese, V., Tylewicz, U., Santagapita, P., Rocculi, P., \& Rosa, M. D. (2012). Isothermal and differential scanning calorimetries to evaluate structural and metabolic alterations of osmo-dehydrated kiwifruit as a function of ripening stage. Innovative Food Science \& Emerging Technologies, 15, 66-71.

Rotta, E. M., Giroux, H. J., Lamothe, S., Bélanger, D., Sabik, H., Visentainer, J. V., \& Britten, M. (2020). Use of passion fruit seed extract (Passiflora edulis Sims) to prevent lipid oxidation in dairy beverages during storage and simulated digestion. Lwt, 123, 109088.

Silva Júnior, J. F., Santiago, Â. M., Galdino, P. O., Santos, N. C., Barros, S. L., de Lima Marsiglia, W. I. M., \& Almeida, R. L. J. (2020). Aplicação da desidratação osmoconvectiva para o aproveitamento tecnológico da casca de banana. Research, Society and Development, 9(1), e103911808-e103911808.

Silva, A. S., \& Pedro, M. A. M. (2018). Estudo da influência da desidratação osmótica na secagem de fatias de abacaxi. Revista Científica, 1(1).

Silva, D. F., Rigueto, C. V. T., Loss, R. A., Guedes, S. F., \& Carvalho, J. W. P. (2017). Tratamento osmótico na obtenção de lascas da polpa de pequi (Caryocar brasiliense) desidratadas. Natural Resources, 7(1), 1-8.

Silva, E. C. O., da Silva, W. P., Gomes, J. P., Silva, C. M. D. P. S., Alexandre, H. V., Farias, V. S. O., \& de Figuiredo, R. M. F. (2019). Drying of albedo and whole peel of yellow passion fruit. Journal of Agricultural Science, 11(6).

Silva, H. W. D., Rodovalho, R. S., Velasco, M. F., Silva, C. F., \& Vale, L. S. (2016). Kinetics and thermodynamic properties related to the drying of'Cabacinha'pepper fruits. Revista Brasileira de Engenharia Agrícola e Ambiental, 20, 174-180.

Souza Neto, M. A. D., Maia, G. A., Lima, J. R., Figueiredo, R. W. D., Souza Filho, M. D. S. M. D., \& Lima, A. D. S. (2005). Desidratação osmótica de manga seguida de secagem convencional: avaliação das variáveis de processo. Ciência e agrotecnologia, 29, 1021-1028.

Zogzas, N. P., Maroulis, Z. B., \& Marinos-Kouris, D. (1996). Moisture diffusivity data compilation in foodstuffs. Drying technology, 14(10), 2225-2253. 